\title{
PROMONOIDAL FUNCTOR CATEGORIES
}

\author{
B. J. DAY
}

(Received 13 May 1976)

\begin{abstract}
In this article a completion process for promonoidal categories is used to determine a sufficient condition for the existence of a promonoidal convolution structure on the category of functors between two promonoidal categories. This, in turn, leads to a partial closed structure on the 2-category of promonoidal categories, promonoidal functors, and promonoidal natural transformations.
\end{abstract}

\section{Introduction}

The purpose of this note is to examine the existence of a promonoidal structure on the category of functors between two promonoidal categories. The 2-category Pmon of promonoidal categories, promonoidal functors, and promonoidal natural transformations (as constructed in Day (1970b)) has certain colimits and a simple symmetric monoidal structure which preserves colimits. Thus, under suitable solution-set conditions, one expects Pmon to be monoidal closed. The author has not yet found such a solution set for the general case but a direct algebraic approach does yield a partial answer under restrictions.

The problem of the existence of the Eilenberg-Moore construction in Pmon was considered in a previous note Day (1977). This problem is related to the present one in that both constructions are, in some sense, inverse limit constructions in Pmon.

For this note we have assumed some knowledge of the elementary definitions and theory (as presented, for example, in Eilenberg and Kelly (1966), Day and Kelly (1969) and Day (1977)). Moreover the calculations may be transferred easily into the "several-objects" version thus yielding analogous results for bicategories of functor categories and probicategories (cf. Benabou (1967) and Day (1974a)). 
Throughout the article we suppose, as usual, that all categorical concepts and algebra are relative to a suitably complete and cocomplete symmetric monoidal closed ground category $\underline{V}=(\underline{V}, \otimes, I, \cdots)$ unless otherwise stated. In particular, we write Pmon in place of $\underline{V}$-Pmon.

\section{Completions of promonoidal categories}

First we recall that a promonoidal category $\underline{A}=(\underline{A}, P, J, \alpha, \rho, \lambda)$ comprises a category $\underline{A}$ and functors $P: \underline{A}^{\mathrm{oP}} \otimes \underline{A}^{\mathrm{oP}} \otimes \underline{A} \rightarrow \underline{V}$ and $J: \underline{A} \rightarrow \underline{V}$ and natural isomorphisms

$$
\begin{aligned}
& \alpha: P(A X-) \circ P\left(A^{\prime} A^{\prime \prime} X\right) \cong P\left(A A^{\prime} X\right) \circ P\left(X A^{\prime \prime}-\right) \\
& \rho: J X \circ P(A X-) \cong \underline{A}(A-) \\
& \lambda: J X \circ P(X A-) \cong \underline{A}(A-)
\end{aligned}
$$

satisfying suitable coherence axioms (namely axioms PC1 and PC2 of Day (1970a)) where "o" denotes profunctor composition. The promonoidal structure has a symmetry $\sigma$ if

$$
\sigma: P\left(A A^{\prime}-\right) \cong P\left(A^{\prime} A-\right)
$$

is a natural isomorphism satisfying axioms PC3 $\left(\sigma^{2}=1\right)$ and PC4 of Day (1970a).

1.1 Remark. We shall not consider the symmetric case explicitly in the sequel, but simply leave it to the reader.

This definition, together with the definitions of promonoidal functor and promonoidal natural transformation are described in Day (1970b) and Day (1977). Thus we obtain the 2-category Pmon whose cocompleteness follows from the fact that "convolution" with $\underline{V}$ (see Day (1970a)) transforms the 2-colimit of a diagram in Pmon into the corresponding limit of monoidal biclosed categories of the form $[\underline{A}, \underline{V}]$, at least if $\underline{V}$ is cartesian closed (see \$).

Similarly we have that two promonoidal categories $\underline{A}$ and $\underline{A}^{\prime}$ have a tensor product $\boldsymbol{A} \otimes \underline{A}^{\prime}$ in Pmon whose components are simply the tensor products of the respective components of $\underline{A}$ and $\underline{A}^{\prime}$; viz,

$$
\begin{gathered}
\bar{P}\left(\left(A, A^{\prime}\right),\left(B, B^{\prime}\right),\left(C, C^{\prime}\right)\right)=P(A B C) \otimes P^{\prime}\left(A^{\prime} B^{\prime} C^{\prime}\right) \\
\bar{J}\left(A, A^{\prime}\right)=J A \otimes J^{\prime} A^{\prime} .
\end{gathered}
$$

Verification of the axioms is straightforward.

From Day (1974b) we recall that each ( $\underline{V}$-small) promonoidal category $\underline{A}$ has a monoidal biclosed completion constructed from the (Isbell-Lambek) 
category completion of a monoidal "approximation" to $\boldsymbol{A}$. More precisely, given $\underline{A}$, consider the Yoneda embedding

$$
\underline{A}^{\mathrm{op}} \subset[\underline{A}, \underline{V}]
$$

and let $\tilde{A}$ denote the full subcategory of $[\underline{A}, \underline{V}]$ determined by the functor $J$ and all finite "paths" $\underline{A}\left(A_{1}-\right) * \cdots * \underline{A}\left(A_{m}-\right)$ of representables (and their isomorphs) under the convolution monoidal structure on $[\underline{A}, \underline{V}]$.

Next form the monoidal biclosed (convolution) category $[\underline{A}, \underline{V}]$ and let $\underline{A}^{0}$ denote the full reflective subcategory of $[\underline{A}, \underline{V}]$ obtained by inverting all the morphisms in $Z^{\circ}$ where

$$
\begin{aligned}
Z^{0}= & \left\{s \in[\underline{\tilde{A}}, \underline{V}]^{2} ; \bar{M}(F * s) \text { and } \bar{M}(s * F)\right. \text { are } \\
& \text { isomorphisms for all } F \in[\underline{\tilde{A}}, \underline{V}]\},
\end{aligned}
$$

and $\bar{M}:[\tilde{A}, \underline{V}] \rightarrow\left[\tilde{A}^{\text {op }}, \underline{V}\right]^{\text {op }}$ is the (Isbell) conjugation functor:

$$
\bar{M} F=\int_{B}[F B, \tilde{A}(-B)] .
$$

The net result is an embedding

$$
E: \underline{A} \rightarrow[\underline{\tilde{A}}, \underline{V}]_{z^{0}}
$$

whose limit-preserving and colimit-preserving properties are discussed in Day (1974b). We note here that $E$ preserves promonoidal structure:

$$
\begin{aligned}
{[\underline{\tilde{A}}, \underline{V}]\left(E A \otimes E A^{\prime}, E A^{\prime \prime}\right) } \\
\quad=[\underline{\tilde{A}}, \underline{V}]\left(\tilde{A}\left(\underline{A}(A-) * \underline{A}\left(A^{\prime}-\right),-\right), \tilde{A}\left(\underline{A}\left(A^{\prime \prime}-\right),-\right)\right) \\
\quad \cong \tilde{A}\left(\underline{A}\left(A^{\prime \prime}-\right), \underline{A}(A-) * \underline{A}\left(A^{\prime}-\right)\right)
\end{aligned}
$$

by the representation theorem,

$$
\cong\left(\underline{A}(A-) * \underline{A}\left(A^{\prime}-\right)\right)\left(A^{\prime \prime}\right)
$$

by the representation theorem,

$$
\cong P\left(A A^{\prime} A^{\prime \prime}\right) \text { for all } A, A^{\prime}, A^{\prime \prime} \in \underline{A} \text {. }
$$

For convenience we also recall the strong trace conditions from Day (1977) $\$ 2$. Given a full embedding $M: \underline{A} \rightarrow \underline{B}$ of a category $\underline{A}$ into a monoidal category $\underline{B}$ (more generally, into a promonoidal category) we ask for conditions under which $\underline{B}$ induces a trace promonoidal structure on $\underline{A}$.

1.2 Proposition. (Strong trace conditions.) The category $\underset{A}{A}$ is promonoidal with respect to the functors: 


$$
\begin{gathered}
P\left(A, A^{\prime}, A^{\prime \prime}\right)=\underline{B}\left(M A \otimes M A^{\prime}, M A^{\prime \prime}\right) \\
J A=\underline{B}(I, M A)
\end{gathered}
$$

if the following canonical morphisms are isomorphisms for all $A, A^{\prime}, A^{\prime \prime} \in \underline{A}$ :

$z_{1}: \underline{B}(I, M X) \circ \underline{B}(M X \otimes M A, M-) \rightarrow \underline{B}(I \otimes M A, M-)$

$z_{1}^{*}: \underline{B}(I, M X) \circ \underline{B}(M A \otimes M X, M-) \rightarrow B(M A \otimes I, M-)$

$z_{2}: \underline{B}\left(M A \otimes M A^{\prime}, M X\right) \circ \underline{B}\left(M X \otimes M A^{\prime \prime}, M-\right)$

$\rightarrow \underline{B}\left(\left(M A \otimes M A^{\prime}\right) \otimes M A^{\prime \prime}, M-\right)$

$z_{2}^{*}: \underline{B}\left(M A^{\prime} \otimes M A^{\prime \prime}, M X\right) \circ \underline{B}(M A \otimes M X, M-)$

$$
\rightarrow \underline{B}\left(M A \otimes\left(M A^{\prime} \otimes M A^{\prime \prime}\right), M-\right) .
$$

If these conditions are satisfied then $M$ becomes a (strong) promonoidal functor.

The proof and examples are given in Day (1977) $\$ 2$ (and also in Day (1970b)).

\section{Promonoidal functor categories}

Suppose $\underline{A}=(\underline{A}, P, J, \cdots)$ and $\underline{B}=(\underline{B}, Q, K, \cdots)$ are small promonoidal categories and, as usual, let $[\underline{A}, \underline{B}]$ denote the category of all functors from $\underline{A}$ to $\underline{B}$; the restriction that $\underline{B}$ be small can usually be waived.

2.1 Remark. The change-of- $\underline{V}$-universe outlined in Day (1973) $\$ 3$ obviates the need to restrict the discussion to Ens-small categories for a fixed Ens.

As shown in Day (1974a), if $\underline{C}$ is a monoidal biclosed category which is suitably complete and cocomplete then the "convolution" of $\underline{A}$ with $\underline{C}$ exists and is described by the formulas:

$$
\begin{gathered}
F * G=\int^{A^{A^{\prime}} P\left(A A^{\prime}-\right) \cdot\left(F A \otimes G A^{\prime}\right)} \\
G / F=\int_{A A^{\prime}}\left[P\left(-A A^{\prime}\right), G A^{\prime} / F A\right] \\
F \backslash G \int_{A A^{\prime}}\left[P\left(A-A^{\prime}\right), F A \backslash G A^{\prime}\right]
\end{gathered}
$$

On taking $C=B^{0}$ the strong trace conditions may be applied to the full embedding

$$
M=[1, E]:[\underline{A}, \underline{B}] \rightarrow\left[\underline{A}, \underline{B}^{\circ}\right] .
$$

Firstly, if the trace structure exists then it is determined by the functors: 
$\left[\underline{A}, \underline{B}^{0}\right]\left(M F \otimes M F^{\prime}, M G\right)$

$$
\begin{aligned}
& =\int_{A} \underline{B}^{0}\left(\left(E F \otimes E F^{\prime}\right) A, E G A\right) \\
& \cong \int_{A} \underline{B}^{0}\left(\int^{X X^{\prime}} P\left(X X^{\prime} A\right) \cdot\left(E F X \otimes E F^{\prime} X^{\prime}\right), E G A\right) \\
& \cong \int_{X X^{\prime} A}\left[P\left(X X^{\prime} A\right), \underline{B}^{0}\left(E F X \otimes E F^{\prime} X^{\prime}, E G A\right)\right] \\
& \cong \int_{X X^{\prime} A}\left[P\left(X X^{\prime} A\right), Q\left(F X, F^{\prime} X^{\prime}, G A\right)\right],
\end{aligned}
$$

this being denoted by $\{P, Q\}\left(F, F^{\prime}, G\right)$, while

$$
\begin{aligned}
{\left[\underline{A}, \underline{B}^{0}\right](I, M F) } & =\int_{A} \underline{B}^{0}(I A, E F A) \\
& \cong \int_{A B^{\prime}}\left[I A\left(B^{\prime}\right), E F A\left(B^{\prime}\right)\right] \\
& \cong \int_{A B^{\prime}}\left[J A \otimes \underline{B}\left(K, B^{\prime}\right), E F A\left(B^{\prime}\right)\right] \\
& \cong \int_{A}[J A, E F A(K)]
\end{aligned}
$$

by the representation theorem; but

$$
\begin{aligned}
E F A(K) & =\underline{\tilde{B}}(\underline{B}(F A,-), K) \\
& \cong K(F A)
\end{aligned}
$$

by the representation theorem. Thus

$$
\left[\underline{A}, \underline{B}^{0}\right](I, M F) \cong \int_{A}[J A, K(F A)]
$$

which is denoted by $\{J, K\}(F)$. By the representation theorem we have

$$
\text { \{rom } \begin{aligned}
\underline{A}, \operatorname{hom} \underline{B}\}(F, G) & =\int_{X A}[\underline{A}(X A), \underline{B}(F X, G A)] \\
& =\int_{A} \underline{B}(F A, G A)
\end{aligned}
$$

which suggests that a uniform notation may be obtained by setting: $P n\left(A_{1} \cdots A_{n}: B\right)=P\left(A_{1} A_{2} X_{1}\right) \circ P\left(X_{1} A_{3} X_{2}\right) \circ \cdots \circ P\left(X_{n-2} A_{n} B\right)$ for $n \geqq 2$ and 


$$
\begin{gathered}
P_{1}(A ; B)=\underline{A}(A B) \\
P_{0}(B)=J B .
\end{gathered}
$$

The strong trace conditions on $M=[1, E]$ now become: $z_{1}:\{J, K\}(G) \circ\{P, Q\}(G, F, H) \cong\{\operatorname{hom} \underset{A}{ }, \operatorname{hom} \underline{B}\}(F, H)$

$z_{1}^{*}:\{J, K\}(G) \circ\{P, Q\}(F, G, H) \cong\{\operatorname{hom} \underline{A}, \operatorname{hom} \underline{B}\}(F, H)$

$z_{2}:\{P, Q\}\left(F, F^{\prime} ; G\right) \circ\{P, Q\}\left(G, F^{\prime \prime} ; H\right) \cong\left\{P_{3}, Q_{3}\right\}\left(F, F^{\prime}, F^{\prime \prime} ; H\right)$

$z_{2}^{*}:\{P, Q\}\left(F^{\prime}, F^{\prime \prime} ; G\right) \circ\{P, Q\}(F, G ; H) \cong\left\{P_{3}, Q_{3}\right\}\left(F, F^{\prime}, F^{\prime \prime} ; H\right)$.

If they are satisfied we denote the resulting structure by $\{\underline{A}, \underline{B}\}$.

It is easily verified that if $\{\underline{A}, \underline{B}\}$ exists then there is an isomorphism

$$
\operatorname{Pmon}\left(\underline{A}^{\prime} \otimes \underline{A}, \underline{B}\right) \cong \operatorname{Pmon}\left(\underline{A}^{\prime},\{\underline{A}, \underline{B}\}\right)
$$

which is 2-natural in $\underline{A}^{\prime} \in$ Pmon. This is an extended form of the exponential law mentioned in Day (1974a) p. 38.

2.2 Remark. On taking $\underline{A}^{\prime}=\underline{I}$ we see that the monoids in $\{\underline{A}, \underline{B}\}$ correspond to the promonoidal functors from $\underline{A}$ to $\underline{B}$.

2.3 Remark. It is clear that if both $\left\{\underline{A}^{\prime} \otimes \underline{A}, \underline{B}\right\}$ and $\{\underline{A}, \underline{B}\}$ exist then $\left\{\underline{A}^{\prime},\{\underline{A}, \underline{B}\}\right\}$ exists with

$$
\left\{\underline{A}^{\prime} \otimes \underline{A}, \underline{B}\right\} \cong\left\{\boldsymbol{A}^{\prime},\{\underline{A}, \underline{B}\}\right\} .
$$

\subsection{ExAmple. (Monoidal functor categories.)}

By the representation theorem the strong trace conditions are satisfied by the embedding $M=[1, E]:[\underline{A}, \underline{B}] \rightarrow\left[\underline{A}, \underline{B}^{\circ}\right]$ if $\left[\underline{A}, \underline{B}^{\circ}\right]\left(M F \otimes M F^{\prime}, M G\right) \cong$ $[\underline{A}, \underline{B}]\left(F \otimes F^{\prime}, G\right)$ and $\left[\underline{A}, \underline{B}^{0}\right](I, M G) \cong[\underline{A}, \underline{B}](I, G)$ for some monoidal structure $(\otimes, I, \cdots)$ on $[\underline{A}, \underline{B}]$. If $\underline{B}$ is monoidal then

$$
\left[\underline{A}, \underline{B}^{0}\right]\left(M F \otimes M F^{\prime}, M G\right)=\left[\underline{A}, \underline{B}^{0}\right]\left(E F \otimes E F^{\prime}, E G\right)
$$

$$
\cong \int_{A X X^{\prime}}\left[P\left(X X^{\prime} A\right), \underline{B}^{0}\left(E F X \otimes E F^{\prime} X^{\prime}, E G A\right)\right]
$$

by definition of $\left\{\underline{A}, \underline{B}^{0}\right\}$,

$$
\cong \int_{A X X^{\prime}}\left[P\left(X X^{\prime} A\right), \underline{B}\left(F X \otimes F^{\prime} X^{\prime}, G A\right)\right]
$$

because $E: \underline{B} \rightarrow \underline{B}^{0}$ preserves the monoidal structure on $\underline{B}$. In particular, this end expression is of the form $[\underline{A}, \underline{B}]\left(F * F^{\prime}, G\right)$ if

$$
\left(F * F^{\prime}\right) A=\int^{X X^{\prime}} P\left(X X^{\prime} A\right) \cdot\left(F X \otimes F^{\prime} X^{\prime}\right)
$$


exists in $\underline{B}$ for all $A \in \underline{A}$ and $F, F^{\prime} \in[\underline{A}, \underline{B}]$. Similarly we have

$$
\begin{aligned}
{\left[\underline{A}, \underline{B}^{0}\right](M I, M G) } & =\int_{A} \underline{B}^{0}(E I A, E G A) \\
& \cong \int_{A} \underline{B}(I A, G A)
\end{aligned}
$$

because $E: \underline{B} \rightarrow \underline{B}^{0}$ preserves the unit object,

$$
=[\underline{A}, \underline{B}](I, G) \text {. }
$$

2.5 EXAMPLE. (Biclosed functor categories.)

If $M=[1, E]:[\underline{A}, \underline{B}] \rightarrow\left[\underline{A}, \underline{B}^{0}\right]$ has the property that

$$
\begin{aligned}
{\left[\underline{A}, \underline{B}^{0}\right]\left(M F \otimes M F^{\prime}, M G\right) } & \cong\left[\underline{A}, \underline{B}^{0}\right]\left(M F^{\prime}, M F \backslash M G\right) \\
& \cong[\underline{A}, \underline{B}]\left(F^{\prime}, F \backslash G\right), \\
{\left[\underline{A}, \underline{B}^{0}\right]\left(M F \otimes M F^{\prime}, M G\right) } & \cong\left[\underline{A}, \underline{B}^{o}\right]\left(M F, M G / M F^{\prime}\right) \\
& \cong[\underline{A}, \underline{B}](F, G / F),
\end{aligned}
$$

and

$$
\left[\underline{A}, \underline{B}^{\circ}\right](M I, M G) \cong[\underline{A}, \underline{B}](I, G)
$$

for some (associative) biclosed structure $(\backslash, /, I, \cdots)$ on $[\underline{A}, \underline{B}]$ then, by the representation theorem, the strong trace conditions are satisfied by $M$.

In particular, suppose $\underline{B}$ is an (associative) biclosed category. Then

$\left[\underline{A}, \underline{B}^{o}\right]\left(M F^{\prime}, M F \backslash M G\right)=\left[\underline{A}, \underline{B}^{0}\right]\left(E F^{\prime} A,(E F \backslash E G)(A)\right)$

$$
\cong \int_{A X X^{\prime}}\left[P\left(X A X^{\prime}\right), \underline{B}^{0}\left(E F^{\prime} A, E F X \backslash E G X^{\prime}\right)\right]
$$

by definition of the biclosed structure on $\left[\underline{A}, \underline{B}^{0}\right]$,

$$
\cong \int_{A X X^{\prime}}\left[P\left(X A X^{\prime}\right), \underline{B}\left(F^{\prime} A, F X \backslash G X^{\prime}\right)\right]
$$

because $E: \underline{B} \rightarrow \underline{B}^{o}$ preserves the biclosed structure on $\underline{B}$ (see [8] $\S 3$ ),

$$
\cong[\underline{A}, \underline{B}]\left(F^{\prime}, F \backslash G\right)
$$

if and only if $\int_{A X X}\left[P\left(X A X^{\prime}\right), B\left(F^{\prime} A, F X \backslash G X^{\prime}\right)\right]$ is of the form $\int_{A} \underline{B}\left(F^{\prime} A,(F \backslash G) A\right)$ for all $F, F^{\prime}, G \in[\underline{A}, \underline{B}]$. In particular this is true if

$$
(F \backslash G) A=\int_{X x^{\prime}}\left[P\left(X A X^{\prime}\right), F X \backslash G X^{\prime}\right]
$$

exists in $\underline{B}$ for all $A \in \underline{A}$ and $F, G \in[\underline{A}, \underline{B}]$. Dually, we obtain a similar assertion for $G / F$. Also 


$$
\begin{aligned}
{\left[\underline{A}, \underline{B}^{0}\right](M I, M G) } & =\int_{A} \underline{B}^{0}(E I A, E G A) \\
& \cong \int_{A} \underline{B}(I A, G A) \\
& =[\underline{A}, \underline{B}](I, G),
\end{aligned}
$$

as in the monoidal case.

Thus, in each of the examples 2.4 and 2.5 , the "convolution" $\{\underline{A}, \underline{B}\}$ exists if suitable colimits or limits exist in $B$ and are preserved by the embedding $E: \underline{B} \rightarrow \underline{B}^{0}$ (see also [7] $\S \S 2-3$ ).

The following example exhibits more clearly the relationship of the general problem of finding promonoidal traces to the local flatness properties of profunctor composition.

2.6 EXAMPLE. ( $\underline{V}$ cartesian closed.)

If we suppose that the monoidal closed structure on $V$ is the cartesian structure then each $\underline{V}$-category $\underline{A}$ has the canonical cocartesian promonoidal structure given by:

$$
\begin{gathered}
P\left(A^{\prime \prime}, A^{\prime}, A\right)=\underline{A}\left(A^{\prime \prime} A\right) \times \underline{A}\left(A^{\prime} A\right) \\
J A=I .
\end{gathered}
$$

If $\underline{B}$ is an arbitrary promonoidal category we can ask when $\{\underline{A}, \underline{B}\}$ exists. This amounts to asking when $\underline{V}$-Pmon, which is canonically a $\underline{V}$-Cat category, admits certain cotensoring over $\underline{V}$-Cat.

To give a (partial) answer to this question we refer back to the strong trace conditions on the embedding $[1, E]:[\underline{A}, \underline{B}] \rightarrow\left[\underline{A}, \underline{B}^{0}\right]$.

2.7 Proposition. ( $V$ cartesian closed.)

If $\boldsymbol{A}$ is taken with the cocartesian promonoidal structure then the embedding $[1, E]:[\underline{A}, \underline{B}] \rightarrow\left[\underline{A}, \underline{B}^{0}\right]$ satisfies the strong trace conditions if and only if the following transformations are isomorphisms for all $F, F^{\prime}, F^{\prime \prime}, H \in[\underline{A}, \underline{B}]$ :

$$
\begin{gathered}
\int^{W} \int_{A}(K W \times Q(W, F A, H A)) \rightarrow \int_{A} \int^{W}(K W \times Q(W, F A, H A)) \\
\int^{W} \int_{A}(K W \times Q(F A, W, H A)) \rightarrow \int_{A} \int^{W}(K W \times Q(F A, W, H A)) \\
\int_{A}^{w}\left(Q\left(F A, F A^{\prime}, W\right) \times Q\left(W, F^{\prime \prime} A, H A\right)\right) \rightarrow \int_{A} \int^{W}\left(Q\left(F A, F^{\prime} A, W\right)\right. \\
\left.\times Q\left(W, F^{\prime \prime} A, H A\right)\right)
\end{gathered}
$$




$$
\begin{aligned}
& \int^{W} \int_{A}\left(Q\left(F^{\prime} A, F^{\prime \prime} A, W\right) \times Q(F A, W, H A)\right) \rightarrow \int_{A} \int^{w}\left(Q\left(F^{\prime} A, F^{\prime \prime} A, W\right)\right. \\
& \times Q(F A, W, H A)) \text {. }
\end{aligned}
$$

Proof. For all $F, F^{\prime}, G \in[\underline{A}, \underline{B}]$ we have:

$$
\begin{gathered}
\{J, K\}(F)=\int_{A}[J A, K F A] \\
=\int_{A}[I, K F A] \\
\cong \lim _{A} K F A . \\
\{P, Q\}\left(F, F^{\prime}, G\right)=\int_{A^{\prime \prime} A^{\prime} A}\left[P\left(A^{\prime \prime}, A^{\prime}, A\right), Q\left(F A^{\prime \prime}, F^{\prime} A^{\prime}, G A\right)\right] \\
=\int_{A^{\prime \prime} A^{\prime} A}\left[\underline{A}\left(A^{\prime \prime} A\right) \times \underline{A}\left(A^{\prime} A\right), Q\left(F A^{\prime \prime}, F^{\prime} A^{\prime}, G A\right)\right] \\
\cong \int_{A} Q\left(F A, F^{\prime} A, G A\right)
\end{gathered}
$$

by the representation theorem applied to $A^{\prime}, A^{\prime \prime} \in \underline{A}$. Similarly

$$
\left\{P_{3}, Q_{3}\right\}\left(F, F^{\prime}, F^{\prime \prime} ; H\right) \cong \int_{A} \int^{W}\left(Q\left(F A, F^{\prime} A, W\right) \times Q\left(W, F^{\prime \prime} A, H A\right)\right) .
$$

Thus, in order to establish the result, it suffices to show that the canonical transformations:

$$
\begin{aligned}
\int^{G}\left(\int_{A}[I, K G A] \times \int_{B} Q(G B, F B, H B)\right) \\
\rightarrow \int^{W} \int_{C}(K W \times Q(W, F C, H C))
\end{aligned}
$$

and

$$
\begin{aligned}
\int^{w} \int_{C}(K W \times Q(W, F C, H C)) \\
\rightarrow \int^{G}\left(\int_{A}[I, K G A] \times \int_{B} Q(G B, F B, H B)\right)
\end{aligned}
$$

are mutually inverse and that the canonical transformations:

$$
\begin{array}{r}
\int^{G}\left(\int_{A} Q\left(F A, F^{\prime} A, G A\right) \times \int_{B} Q\left(G B, F^{\prime \prime} B, H B\right)\right) \\
\rightarrow \int^{w} \int_{C}\left(Q\left(F C, F^{\prime} C, W\right) \times Q\left(W, F^{\prime \prime} C, H C\right)\right)
\end{array}
$$


and

$$
\begin{aligned}
\int^{W} \int_{C}\left(Q\left(F C, F^{\prime} C, W\right) \times Q\left(W, F^{\prime \prime} C, H C\right)\right) \\
\rightarrow \int^{G}\left(\int_{A} Q\left(F A, F^{\prime} A, G A\right) \times \int_{B} Q\left(G B, F^{\prime \prime} B, H B\right)\right)
\end{aligned}
$$

are mutually inverse. This is a straightforward exercise, the transpose arguments yielding the transpose isomorphism conditions.

Thus, if $\underline{\boldsymbol{A}}$ is finite, $\{\underline{\boldsymbol{A}}, \boldsymbol{B}\}$ exists if $\int^{\boldsymbol{G}}$ is "sufficiently exact". For example if $\underline{V}=$ Ens and $\underline{A}$ is finite then $\{\underline{A}, \underline{B}\}$ exists if the opposite of the twisted-arrow category* of $\underline{B}$ is filtered.

*REMARK. Here the twisted-arrow category $\underline{B}_{*}$ of $\underline{B}$ is the category whose objects are the morphisms $f: B \rightarrow B^{\prime}$ of $\underline{B}$ and whose morphisms $(h, k): f \rightarrow f^{\prime}$ are the pairs $h: B^{\prime \prime} \rightarrow B$ and $k: B^{\prime} \rightarrow B^{\prime \prime \prime}$ of morphisms in $\underline{B}$ such that $f^{\prime}=k f h$ (as defined in Mac Lane (1971) IX §6). In practice it seems easier to see when $\underline{B}_{*}$ is filtered (e.g. if $\underline{B}$ is finitely complete) than it is to see when $\underline{B}_{*}^{\text {op }}$ is filtered.

\section{A comparison theorem}

If $\underline{B}$ is a sufficiently complete and cocomplete monoidal biclosed category then $\underline{B}^{\circ}$ is category equivalent to the standard Isbell-Lambek completion $\underline{B}^{*}$ of $\underline{B}$ (see Day (1973) $\S 2$ ). This leads to the equivalence (see Day (1974a) §7):

$$
\left\{\underline{A}, \underline{B}^{\circ}\right\} \cong\{\underline{A}, \underline{B}\}^{0}
$$

This comparison is slightly extended in this section.

As in Day (1973) §3, consider the following diagram in which $\bar{N} \multimap\left[N^{o p}, 1\right]$ and $\underline{W}$ is a sufficiently large " $\underline{V}$-universe" to make $[\underline{B}, \underline{V}]$ "small".

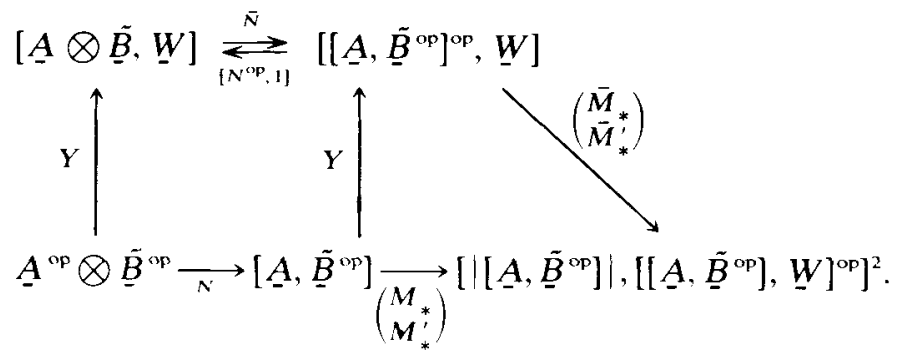


We suppose here that the dense (= adequate) Yoneda functor:

$$
N: \underline{A}^{\text {op }} \otimes \underline{B}^{\text {op }} \rightarrow\left[\underline{A}, \underline{B}^{\text {op }}\right] ; N\left(A B^{\prime}\right)=\underline{A}(A-) \cdot B^{\prime}
$$

exists and, in addition, we suppose that $\left[\underline{A}, \underline{\tilde{B}}^{\text {op }}\right]$ is monoidal by virtue of the monoidal structure of $\tilde{B}^{\text {op }}$ (see 2.4 Example). The functor $\bar{M}_{*}$ (respt. $\bar{M}_{*}^{\prime}$ ) is the left Kan extension of $M_{*}$ (respt. $M_{*}^{\prime}$ ) along $Y$ where:

$$
\begin{aligned}
M_{*}(H)_{F} & =\left[\underline{A}, \tilde{\tilde{B}}^{\mathrm{op}}\right](F * H,-) \\
M_{*}^{\prime}(H)_{F} & =\left[\underline{A}, \tilde{\hat{B}}^{\mathrm{op}}\right](H * F,-) .
\end{aligned}
$$

Thus

$$
\bar{M}_{*}(G)_{F} \cong \int_{F^{\prime}}\left[\left(\left[\underline{A}, \underline{B}^{\mathrm{op}}\right](-, F) * G\right)\left(F^{\prime}\right),\left[\underline{A}, \underline{B}^{\mathrm{op}}\right]\left(F^{\prime},-\right)\right]
$$

and

$$
\bar{M}_{*}^{\prime}(G)_{F} \cong \int_{F^{\prime}}\left[\left(G *\left[\underline{A}, \underline{\tilde{B}}^{\mathrm{op}}\right](-, F)\right)\left(F^{\prime}\right),\left[\underline{A}, \underline{\tilde{B}}^{\mathrm{op}}\right]\left(F^{\prime},-\right)\right] .
$$

By [6] $\$ 2$ (Lemma 2.1) the category of fractions of $\left[\left[\underline{A}, \underline{B}^{\text {op }}\right]^{\text {op }}, \underline{W}\right]$ with respect to the class of morphisms inverted by both $\bar{M}_{*}$ and $\bar{M}_{*}^{\prime}$ is equivalent to the monoidal closure $\left\{\underline{A}, \underline{B}^{\text {op }}\right\}^{0}$ of the Isbell-Lambeck completion of $\left[\underline{A}, \underline{\tilde{B}}^{\text {op }}\right]$.

We now ask when $\left\{\underline{A}, \underline{B}^{\text {op }}\right\}^{0}$ is category equivalent to the category of fractions of $[\underline{A} \otimes \underline{B}, \underline{V}]$ with respect to the class of morphisms inverted by both of the functors $\bar{M}_{*} \bar{N}$ and $\bar{M}_{*}^{\prime} \bar{N}$. By Day (1973) Corollary 3.2, this is equivalent to requiring that the canonical transformations

$$
M_{*}(H)_{F} \rightarrow \int_{A B^{\prime}}\left[\left[\underline{A}, \underline{B}^{\text {op }}\right]\left(N\left(A B^{\prime}\right), H\right), M_{*} N\left(A B^{\prime}\right)\right]_{F}
$$

and

$$
M_{*}^{\prime}(H)_{F} \rightarrow \int_{A B^{\prime}}\left[\left[\underline{A}, \underline{B}^{\text {op }}\right]\left(N\left(A B^{\prime}\right), H\right), M_{*}^{\prime} N\left(A B^{\prime}\right)\right]_{F}
$$

be isomorphisms for all $F, H \in\left[\underline{A}, \underline{B}^{\text {op }}\right]$. By the representation theorem we have:

$$
\begin{aligned}
& \int_{A B^{\prime}} {\left[\int_{A^{\prime}} \underline{\tilde{B}}^{\mathrm{op}}\left(\underline{A}\left(A A^{\prime}\right) \cdot B^{\prime}, H A^{\prime}\right),\left[\underline{A}, \underline{\tilde{B}}^{\mathrm{op}}\right]\left(F * N\left(A B^{\prime}\right),-\right)\right] } \\
& \cong \int_{A B^{\prime}}\left[\underline{\tilde{B}}^{\mathrm{op}}\left(B^{\prime}, H A\right),\left[\underline{A}, \underline{\tilde{B}}^{\mathrm{op}}\right]\left(F * N\left(A B^{\prime}\right),-\right)\right] \\
& \cong \int_{A}\left[\underline{A}, \underline{\tilde{B}}^{\mathrm{op}}\right](F * N(A, H A),-) .
\end{aligned}
$$


This is of the form $M_{*}(H)_{F}=\left[A, \underline{B}^{\text {op }}\right](F * H,-)$ if and only if

$$
\int^{A} F * N(A, H A) \cong F * H
$$

for all $F, H \in\left[\underline{A}, \underline{B}^{\text {op }}\right]$. Similarly $\int_{A}\left[\underline{A}, \underline{B}^{\text {op }}\right](N(A, H A) * F,-)$ is of the form $M_{*}^{\prime}(H)_{F}=\left[\underline{A}, \underline{\tilde{B}}^{\text {op }}\right](H * F,-)$ if and only if

$$
\int^{A} N(A, H A) * F \cong H * F
$$

for all $F, H \in\left[\underline{A}, \underline{B}^{\circ p}\right]$. In particular, these requirements are satisfied if $F *-$ and $-* F$ preserve tensoring or, more strongly, if $\left[\underline{A}, \underline{\tilde{B}}^{\text {op }}\right]$ is monoidal biclosed.

It remains to describe the category $[\underline{A} \otimes \underline{B}, \underline{W}]_{\Sigma}, \Sigma=\left\{\sigma ; \bar{M}_{*} \bar{N}(\sigma)\right.$ and $\bar{M}_{*}^{i} \bar{N}(\sigma)$ are isomorphisms $\}$, and compare it with $\left\{\underline{A}, \underline{B}^{\circ}\right\}$. We have

$$
\begin{aligned}
\bar{N}(\sigma) & =\int^{A B^{\prime}} \sigma\left(A B^{\prime}\right) \cdot Y N\left(A B^{\prime}\right) \\
& =\int^{A B^{\prime}} \sigma\left(A B^{\prime}\right) \cdot\left[\underline{A}, \underline{B}^{o p}\right]\left(-, N\left(A B^{\prime}\right)\right)
\end{aligned}
$$

therefore, by the representation theorem, we have:

$$
\begin{aligned}
& \left(\bar{M}_{*} \bar{N} \sigma\right)_{F}=\int_{F^{\prime}}\left[\left[\underline{A}, \underline{B}^{\mathrm{op}}\right](-, F) * \int^{A B^{\prime}} \sigma\left(A B^{\prime}\right) \cdot\left[\underline{A}, \tilde{B}^{\mathrm{op}}\right]\left(-, N\left(A B^{\prime}\right)\right)\left(F^{\prime}\right),\right. \\
& \left.\left[\underline{A}, \hat{B}^{\mathrm{op}}\right]\left(F^{\prime},-\right)\right] \\
& \cong \int_{F^{\prime} A B^{\prime}}\left[\sigma\left(A B^{\prime}\right),\left[\left[\underline{A}, \underline{B}^{\mathrm{op}}\right]\left(F^{\prime}, F * N\left(A B^{\prime}\right)\right),\left[\underline{A}, \underline{\tilde{B}}^{\mathrm{op}}\right]\left(F^{\prime},-\right)\right]\right. \\
& \approx \int_{A B^{\prime}}\left[\sigma\left(A B^{\prime}\right),\left[\underline{A}, \underline{\tilde{B}}^{\mathrm{op}}\right]\left(F * N\left(A B^{\prime}\right),-\right)\right]
\end{aligned}
$$

for all $F \in\left[\underline{A}, \underline{\tilde{B}}^{\text {op }}\right]$ and, similarly,

$$
\left(\bar{M}_{*}^{\prime} \bar{N} \sigma\right)_{F}=\int_{A B^{\prime}}\left[\sigma\left(A B^{\prime}\right),\left[\underline{A}, \underline{\tilde{B}}^{\mathrm{op}}\right]\left(N\left(A B^{\prime}\right) * F,-\right)\right]
$$

for all $F \in\left[\underline{A}, \underline{B}^{\text {op }}\right]$.

By definition of $\underline{B}^{0}$ the category $\left\{\underline{A}, \underline{B}^{0}\right\}$ is characterised as the category of fractions of $[\underline{A} \otimes \underline{\tilde{B}}, \underline{W}] \cong[\underline{A},[\underline{\tilde{B}}, \underline{W}]]$ with respect to the class of morphisms inverted by the functor:

$$
\left(\begin{array}{l}
S_{*} \\
S_{*}^{\prime}
\end{array}\right):[\underline{A},[\underline{\tilde{B}}, W]] \rightarrow\left[\underline{A},\left[|\underline{\tilde{B}}|,\left[\underline{\tilde{B}}^{\mathrm{op}}, \underline{W}\right]\right]^{2}\right]
$$

described by: 


$$
\begin{aligned}
S_{*}(\sigma(A-))_{B^{\prime}} & =\int_{B^{\prime \prime}}\left[\left(\sigma(A-) * \underline{\tilde{B}}\left(B^{\prime}-\right)\right) B^{\prime \prime}, \underline{\tilde{B}}\left(-B^{\prime \prime}\right)\right] \\
& \cong \int_{B^{\prime \prime}}\left[\int^{x^{\prime}} \sigma\left(A X^{\prime}\right) \otimes \underline{B}\left(B^{\prime} * X^{\prime}, B^{\prime \prime}\right), \underline{\tilde{B}}\left(-B^{\prime \prime}\right)\right]
\end{aligned}
$$

by definition of “*”,

$$
\cong \int_{X^{\prime}}\left[\sigma\left(A X^{\prime}\right), \underline{B}\left(-, B^{\prime} * X^{\prime}\right)\right]
$$

by the representation theorem, and, similarly,

$$
S^{\prime}(\sigma(A-))_{B^{\prime}} \cong \int_{X^{\prime}}\left[\sigma\left(A X^{\prime}\right), \underline{\tilde{B}}\left(-, X^{\prime} * B^{\prime}\right)\right]
$$

Thus, on comparing $[\underline{A} \otimes \underline{B}, \underline{W}]_{\Sigma}$ with $\left\{\underline{A}, \underline{B}^{0}\right\}$, we see immediately that $[\underline{A} \otimes \underline{B}, \underline{W}]_{\Sigma}$ is reflectively embedded in $\left\{\underline{A}, \underline{B}^{\circ}\right\}$ by a monoidal biclosed reflection. Thus, if (3.2) and (3.2) are satisfied then $\left\{\underline{A}, \underline{B}^{\text {op }}\right\}^{0}$ is monoidally reflectively embedded in $\left\{\underline{A}, \underline{B}^{\circ}\right\}$.

If we now assume, in addition to requiring that the monoidal structure on $\tilde{B}^{\text {op }}$ commute with the requisite colimits, that $\underline{B}^{\text {op }}$ is sufficiently cotensored then the above mentioned reflective embedding is a category equivalence. In order to verify this suppose that

$$
\int_{A B^{\prime}}\left[\sigma\left(A B^{\prime}\right),\left[\underline{A}, \underline{\tilde{B}}^{\mathrm{op}}\right]\left(F * N\left(A B^{\prime}\right), F^{\prime}\right)\right]
$$

is an isomorphism for all $F, F^{\prime} \in\left[\underline{A}, \underline{\tilde{B}}^{\text {op }}\right]$. Then we may choose $F=N\left(A^{\prime} B^{\prime \prime}\right)$ and $F^{\prime}=\left[\underline{A}\left(-A^{\prime \prime}\right), B^{\prime \prime \prime}\right]$ to ensure that

$$
\int_{A B^{\prime}}\left[\sigma\left(A B^{\prime}\right),\left[P\left(A^{\prime} A A^{\prime \prime}\right), \underline{B}^{\text {op }}\left(B^{\prime \prime} * B^{\prime}, B^{\prime \prime \prime}\right)\right]\right]
$$

is an isomorphism for all $A^{\prime}, A^{\prime \prime} \in A$ and all $B^{\prime \prime}, B^{\prime \prime \prime} \in \underline{B}^{\text {op }}$. Because, by the axioms for promonoidal structure,

$$
\int^{A^{\prime}} J A^{\prime} \otimes P\left(A^{\prime} A A^{\prime \prime}\right) \cong \underline{A}\left(A A^{\prime \prime}\right)
$$

we have, by the representation theorem, that

$$
\int_{B^{\prime}}\left[\sigma\left(A^{\prime \prime} B^{\prime}\right), \tilde{B}^{\mathrm{op}}\left(B^{\prime \prime} * B^{\prime}, B^{\prime \prime \prime}\right)\right]
$$

is an isomorphism for all $A^{\prime \prime} \in \underline{A}$ and $B^{\prime \prime}, B^{\prime \prime \prime} \in \underline{B}^{\text {op}}$, as required; the dual (= transpose) argument gives the result with $B^{\prime} * B^{\prime \prime}$ replacing $B^{\prime \prime} * B^{\prime}$ here. 


\section{Appendix; colimits in Pmon}

From the fact that the convolution process transforms the objects of Pmon into monoidal biclosed structures of the form $\{\underline{A}, \underline{V}\}$ we may deduce something of the existence of colimits in Pmon.

4.1 Proposition. Pmon has all small-indexed coproducts and these are preserved by the forgetful functor Pmon $\rightarrow$ Cat.

Proof. If $\left\{A_{\lambda} ; \lambda \in \Lambda\right\}$ is a set of promonoidal categories we form $\Sigma \underline{A}_{\lambda}$ in Cat and give it the structure induced by the isomorphism:

$$
\left[\sum \underline{A}_{\lambda}, \underline{V}\right] \cong \prod\left\{\underline{A}_{\lambda}, \underline{V}\right\}
$$

We then have:

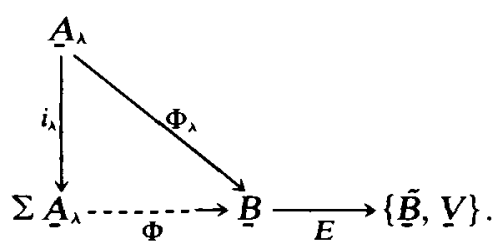

To see that Pmon $\left(\sum \underline{A}_{\lambda}, \underline{B}\right) \cong \Pi \operatorname{Pmon}\left(\underline{A}_{\lambda}, \underline{B}\right)$ for all $\underline{B} \in$ Pmon note that the following diagram commutes for all $\underline{B} \in$ Pmon:

$$
\begin{aligned}
\operatorname{Pmon}\left(\sum \underline{A}_{\lambda},\{\tilde{B}, \underline{V}\}\right) & =\prod \operatorname{Pmon}\left(\underline{A}_{\lambda},\{\underline{\tilde{B}}, \underline{V}\}\right) \\
\operatorname{Pmon}\left(\underline{\tilde{B}},\left\{\sum \underline{A}_{\lambda}, \underline{V}\right\}\right) & \rightarrow \prod \operatorname{Pmon}\left(\underline{\tilde{B}},\left\{\underline{A}_{\lambda}, \underline{V}\right\}\right) .
\end{aligned}
$$

It is straightforward to check that the $\otimes$ on Pmon preserves coproducts. In a similar manner we can ask whether Pmon is tensored as a 2-category. One checks easily that if $\underline{V}$ is cartesian closed and $\underline{A}$ is a category with the cocartesian promonoidal structure (described in 2.6 Example) then $\underline{A} \cdot \underline{B}$ exists for all $\underline{B} \in$ Pmon. Moreover this tensoring is preserved by the $\otimes$ on Pmon.

We now turn to the question of coequalisers in Pmon. Suppose

$$
\stackrel{\oplus}{\stackrel{\Phi}{\rightleftharpoons}} \underset{\sim}{\longrightarrow} \stackrel{\Gamma}{\longrightarrow} C
$$

is a coequaliser diagram in Pmon.

4.2 Proposition. If the functors 


$$
\{\Phi, 1\},\{\Psi, 1\}:\{\underline{B}, \underline{V}\} \rightarrow\{\underline{A}, \underline{V}\}
$$

are strict monoidal functors then the coequaliser in Pmon of $\Phi$ and $\Psi$ exists and is preserved by the forgetful functor from Pmon to Cat.

Proof. Let $\gamma: \underline{B} \rightarrow \underline{C}$ be the coequaliser of $\phi$ and $\psi$ in Cat. Because $\{\Phi, 1\}$ and $\{\Psi, 1\}$ are strict monoidal functors their equaliser exists as the monoidal category structure on $[\underline{C}, \underline{V}]$ induced by that on $\{\underline{B}, \underline{V}\}$; to see that this structure is biclosed it suffices to note that $[\Gamma, 1]$ preserves monoidal structure and colimits and is, in fact, monadic over $\{\underline{B}, \underline{V}\}$ because $\gamma$ is surjective on objects. The remainder of the verification is as for coproducts.

4.3 PROPOSITION. The monoidal structure on Pmon preserves:

(i) coequalisers if it preserves epimorphisms.

(ii) those coequalisers preserved by $\mid$ ? : Pmon $\rightarrow$ Ens.

Proof. Let

$$
\underset{\psi}{\stackrel{\oplus}{\rightleftarrows}} \underline{B} \stackrel{\Gamma}{\longrightarrow} C
$$

be a coequaliser diagram in Pmon. Let $\underline{K} \in$ Pmon and $H: \underline{B} \otimes \underline{K} \rightarrow \underline{D}$ be a promonoidal functor such that $H(\Phi \otimes 1)=H(\Psi \otimes 1)$. Let $E: \underline{D} \rightarrow\{\underline{D}, \underline{V}\}$ be the evaluation embedding of $\underline{D}$.

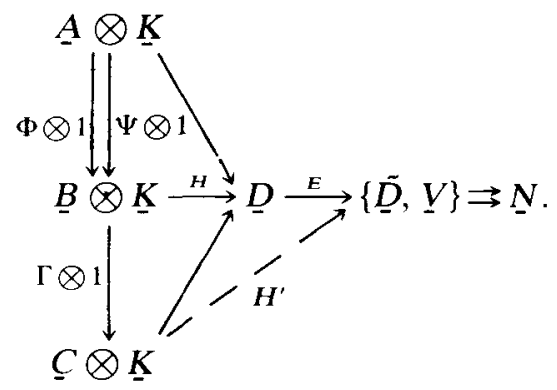

Because $E H(\Phi \otimes 1)=E H(\Psi \otimes 1, E H$ induces a promonoidal functor $H^{\prime}: \subseteq \otimes \underline{K} \rightarrow\{\underline{D}, \underline{V}\}$. For (i) we use the fact that $E$ is the equaliser of its cokernel pair $N$ in Cat. For (ii) we use the fact that $\gamma$ is a surjection on objects to factor $H^{\prime}$ through $E$ (uniquely).

With regard to the preceding propositions we also have:

4.4 Proposition. If the diagram 


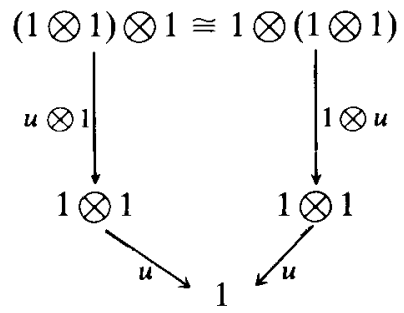

is a pushout diagram in $\underline{V}$ and $u: 1 \otimes 1 \rightarrow 1$ is an epimorphism in $\underline{V}$ then the functor $|?|: \underline{V}$-Pmon $\rightarrow$ Ens has a right adjoint.

Proof. We define $G:$ Ens $\rightarrow \underline{V}$-Pmon by taking $G X$ to be the $\underline{V}$ category whose object set is $X$ and such that $G X(A, B)=1, P(A, B, C)=1$, and $J A=1$ for all $A, B, C \in X$. The hypotheses on $u: 1 \otimes 1 \rightarrow 1$ imply that if $\underline{A}$ is a $\underline{V}$-category and $S: \underline{A}^{\mathrm{op}} \rightarrow \underline{V}$ and $T: \underline{A} \rightarrow \underline{V}$ are the constant functors to 1 then $\int^{A} S A \otimes T A=1$. The axiom verifications for $G X$ are then straightforward.

Clearly the hypotheses on $u: 1 \otimes 1 \rightarrow 1$ are satisfied if $1 \otimes 1=1$; in particular if $I$ is terminal in $\underline{V}$.

4.5 COROLlaRY. If the hypotheses of the preceding proposition are satisfied in $\underline{V}$ then each coequaliser diagram $\underline{A} \rightrightarrows \underline{B} \rightarrow \underline{C}$ in $\underline{V}$-Pmon is transformed into an equaliser diagram of the form $\{\underline{C}, \underline{V}\} \rightarrow\{\underline{B}, \underline{V}\} \rightrightarrows\{\underline{A}, \underline{V}\}$ by convolution.

\section{Bibliography}

J. Bénabou (1967), 'Introduction to bicategories', Reports of the Midwest Category Seminar I (Springer Lecture Notes, Vol. 47), 1-77.

F. Borceux and G. M. Kelly (1975), 'A notion of limit for enriched categories', Bull. Austral. Math. Soc. 12, 49-72.

B. J. Day and G. M. Kelly (1969), 'Enriched functor categories', Reports of the Midwest Category Seminar III (Springer Lecture Notes, Vol. 106), 178-191

B. J. Day (1970a), 'On closed categories of functors', Reports of the Midwest Category Seminar IV (Springer Lecture Notes, Vol. 137), 1-38.

B. J. Day (1970b), 'Construction of biclosed categories', Ph.D. thesis, University of New South Wales.

B. J. Day (1973), 'Note on monoidal localisation', Bull. Austral. Math. Soc., 8, 1-16.

B. J. Day (1974a), 'On closed categories of functors II', Category Seminar, Sydney 1972/73 (Springer Lecture Notes, Vol. 420), 20-53.

B. J. Day (1974b), 'An embedding theorem for closed categories', Category Seminar, Sydney 1972/73 (Springer Lecture Notes, Vol. 420), 55-64.

B. J. Day (1977), 'Note on monoidal monads', J. Austral. Math. Soc. (Ser. A) 23, $292-311$.

E. Dubuc (1970), Kan extensions in enriched category theory (Springer Lecture Notes, Vol. 145). 
S. Eilenberg and G. M. Kelly (1966), 'Closed categories', Proc. Conference on Categorical Algebra, La Jolla, 1965 (Springer-Verlag), 421-562.

A. Kock (1971), 'Closed categories generated by commutative monads', J. Austral. Math. Soc. 12, 405-424.

S. Mac Lane (1971), Categories for the working mathematician (GTM5, Springer-Verlag, New York, Heidelberg, Berlin).

R. H. Street (1976), 'Limits indexed by category-valued 2-functors'; to appear in J. Pure and Applied Algebra, Vol. 10.

Department of Pure Mathematics,

University of Sydney,

N.S.W. 2006,

Australia. 